Sciences Sociales et Santé, Vol. 30, $n^{\circ} 1$, mars 2012

\title{
Le traitement adjuvant du cancer du sein par tamoxifène. Entre risques et bénéfices thérapeutiques
}

\author{
Aline Sarradon-Eck*, Isabelle Pellegrini***
}

\begin{abstract}
Résumé. La «balance risque-bénéfice » est l'un des piliers du processus décisionnel biomédical conduisant à choisir une thérapeutique à administrer. Cet article se propose d'éclairer, en le contextualisant, le processus décisionnel concernant le traitement de patientes atteintes d'un cancer du sein. Par une approche anthropologique descriptive de la prescription d'un traitement adjuvant par tamoxifène visant à réduire le risque de récidive, il explore les décalages entre l'appréhension des risques et des bénéfices du traitement par les patientes et par les médecins, et il analyse les logiques cognitives, symboliques et sociales qui les sous-tendent. Les représentations des femmes sont relatives au cancer (peur de la maladie), à ses traitements (efficacité) et au tamoxifène (peur des hormones et du vieillissement); elles peuvent conduire à une hiérarchisation symbolique des traitements. Les médecins, quant à eux, obéissent à une logique pro-
\end{abstract}

\footnotetext{
* Aline Sarradon-Eck, anthropologue, Centre Norbert Elias, Équipe GReCSS/PAS Anthropologie de la Santé, MMSH, 5, rue du Château de l'Horloge, BP 647, 13094 Aix-en-Provence, France ; aline.sarradon@wanadoo.fr

** Isabelle Pellegrini psychologue clinicienne, INSERM, UMR 912 (SE4S), Institut Paoli-Calmette, 232, boulevard Sainte-Marguerite, 13273 Marseille Cedex 09, France.
} 
fessionnelle d'intervention dirigée préférentiellement vers la prévention de la récidive.

Mots-clés : cancer, adhésion thérapeutique, relation médecin-malade, calcul risque-bénéfice.

La participation des malades à la décision thérapeutique les concernant est devenue une norme médicale. Sa justification est, entre autres, éthique : elle s'appuie sur le principe reconnu par la médecine du respect de l'autonomie du patient. Elle est renforcée par l'appareil législatif avec, en France, la loi relative aux droits du malade et à la qualité du système de santé du 4 mars 2002. Selon la loi, le patient doit être informé des bénéfices et des risques des traitements proposés pour qu'il prenne, avec le médecin, les décisions concernant sa santé.

Un des piliers du processus décisionnel biomédical conduisant à choisir la thérapeutique à administrer est la "balance risque-bénéfice ». Ce schéma décisionnel consistant à faire un choix guidé par le calcul des avantages et des inconvénients d'un traitement à administrer au malade est une part irréductible de la biomédecine. Il repose sur une rationalité fondée sur la logique économique coût-bénéfice dont la position est hégémonique dans le champ de la santé et de la maladie (Massé, 1997).

Sans remettre en cause le présupposé théorique que les patients sont des acteurs rationnels, l'anthropologie s'efforce de révéler «les processus décisionnels qui ne respectent généralement pas la logique utilitariste » (Massé, 1997 : 59) et de mettre au jour la pluralité des logiques qui régissent les choix des individus dans le domaine des thérapies comme dans d'autres domaines socialement situés.

Dans cet article, nous proposons d'éclairer le processus décisionnel concernant un traitement de patientes atteintes d'un cancer du sein en le contextualisant. Il s'agit de montrer les logiques symboliques sous-jacentes aux choix des patientes, de décoder les significations et réinterprétations du médicament, de constater les contraintes organisationnelles pesant sur ces choix et de souligner les rôles sociaux et les rapports de pouvoir véhiculés par le médicament (Desclaux et Lévy, 2003).

L'article porte sur la prescription d'un traitement adjuvant par tamoxifène. Un traitement est considéré comme adjuvant lorsqu'il est donné en prévention des récidives, après le traitement qui enlève ou détruit la tumeur. Le tamoxifène est donné aux femmes dont la tumeur contient des récepteurs hormonaux positifs dans le but de réduire leur 
risque de récidive (1). Le tamoxifène est un médicament facile à prendre (un comprimé par jour pendant cinq ans). Il comporte cependant de rares effets à potentiel létal (thrombose veineuse, cancer de l'endomètre) et de nombreux effets indésirables liés à la privation œstrogénique (2). Selon les standards médicaux des centres de lutte contre le cancer où nous avons conduit notre enquête de terrain, le tamoxifène est prescrit à la fin du parcours institutionnel de soins (associant chirurgie, radiothérapie et/ou chimiothérapie) pendant cinq ans lorsque la patiente n'est pas ménopausée. Les femmes en périménopause reçoivent du tamoxifène pendant deux ans, puis le relais est pris par les antiaromatases, un traitement similaire quant à ses objectifs antihormonaux mais plus adapté (en termes d'efficacité) aux femmes ménopausées.

La prescription de tamoxifène en adjuvant correspond à une triple incertitude : le médicament est prescrit à des femmes qui pourraient rechuter ; son efficacité sur la prévention de la récidive est partielle ; son innocuité à court et moyen terme est loin d'être parfaite. La décision des femmes de prendre ou non ce médicament, et celle des médecins de l'administrer, est une porte d'entrée intéressante pour explorer, selon une approche qui se veut résolument descriptive, les dimensions cognitives, symboliques et sociales (rapports médecins-malades) de l'anticipation des bénéfices et des représentations du risque en balance.

\section{Méthodologie}

L'analyse s'appuie sur une enquête de terrain réalisée en 2006 et 2007 dans deux centres de lutte contre le cancer (Nice et Marseille). Bien que le corpus principal de la recherche soit constitué d'entretiens avec des patientes soignées pour un cancer du sein auxquelles un traitement par tamoxifène en adjuvant a été proposé, l'enquête ethnographique conduite comporte classiquement plusieurs phases et techniques de recueil de données. Une phase d'imprégnation et d'observation flottante nous a permis

(1) Administré pendant cinq ans, le tamoxifène réduirait de moitié le risque de rechute et d'un tiers la mortalité à 15 ans (Early Breast Cancer Trialists' Collaborative Group, 2005).

(2) Le tamoxifène est un modulateur spécifique du récepteur aux œstrogènes. Dans les cancers du sein hormono-dépendants, il agit principalement comme antagoniste des œstrogènes au niveau du tissu mammaire et comme agoniste partiel dans d'autres tissus cibles, ce qui explique la survenue d'effets indésirables (Clarke et al., 2001). 
de mieux comprendre le contexte d'énonciation de la parole des patientes rencontrées, leur vécu de la maladie et des traitements (3). Nous avons observé différents moments et lieux de la prise en charge tout au long du parcours de patientes atteintes de cancer du sein et rencontré diverses catégories de professionnels impliqués dans cette prise en charge. Dans une approche inductive, nous avons observé des lieux où se joue la décision médicale concernant l'hormonothérapie adjuvante : des réunions de concertation pluridisciplinaires sur le cancer du sein (3 à Nice, 2 à Marseille) et des consultations en amont du traitement (annonce de la maladie, remise du programme personnalisé de soins) et pendant le traitement (première prescription de tamoxifène, renouvellement du traitement et consultation de suivi) - au total, 111 consultations (de chirurgiens, oncologues et radiothérapeutes) ont été observées. Dans un objectif de triangulation (Olivier de Sardan, 2008), nous avons analysé des « conversations » se rapportant au tamoxifène sur le forum de discussion de la Ligue contre le cancer (4). Les matériaux analysés privilégient la parole des patientes au travers des entretiens accordés aux chercheuses. Ils ont été conduits auprès de 34 patientes (5) pouvant bénéficier d'une hormonothérapie adjuvante (6) et âgées de 35 à 65 ans lors du premier entretien (Encadré 1). Des entretiens réitérés (2 à 3 par femme) ont été réalisés à chaque fois que cela a été possible, de façon à cerner le plus finement possible le caractère dynamique du vécu du traitement par les patientes ; au total, 53 entretiens ont été réalisés et analysés. De même, nous avons cherché à rencontrer des femmes à tous les temps du traitement ainsi que des femmes ayant refusé ou interrompu le traitement, des femmes ménopausées, périménopausées ou non ménopausées au moment du diagnostic.

(3) Nous avons été présentées aux patientes et aux professionnels de santé comme des chercheuses (anthropologue pour l'une, psychologue pour la seconde) effectuant une recherche sur le suivi du traitement par tamoxifène. Il s'agissait d'une observation non participante. Nous ne portions pas de blouse blanche afin de ne pas être assimilées à l'équipe soignante, ce qui dans l'un des centres n'est pas forcément distinctif puisque la plupart des médecins ne portent pas de blouse lors des consultations.

(4) Une « conversation » comporte un message initial (question, témoignage) et les réponses des internautes inscrits sur le forum, ainsi que la réponse du médecin de la Ligue lorsque celui-ci est interpellé. Certaines conversations comportent jusqu'à huit messages envoyés.

(5) L'effectif a été déterminé en fonction des premiers résultats d'analyse et du phénomène de saturation de l'information (Olivier de Sardan, 1995).

(6) Nous n'avons pas eu accès aux caractéristiques histologiques tumorales ; cependant, les femmes rencontrées étaient toutes considérées comme en « rémission ». 


\section{Encadré 1 \\ Quelques caractéristiques du groupe des répondantes $(n=34)$}

Les répondantes étaient âgées de 35 à 64 ans ; 25 étaient mariées ou vivaient en couple ; 19 étaient en activité professionnelle au moment de l'entretien (dont 5 en mi-temps thérapeutique), 15 étaient sans activité (retraite : 4 , congé longue maladie : 3 , invalidité : 1 , congé maternité : 1 , sans emploi : 6). Au moment du diagnostic, 20 femmes n'étaient pas ménopausées, 7 étaient en périménopause et 7 étaient ménopausées. Sur la base de leur déclaration (nous n'avons pas eu accès aux dossiers médicaux), 9 femmes avaient une tumeur de stade 1 (absence de ganglions axillaires positifs), 4 avaient une tumeur de stade 2 (présence de ganglions axillaires positifs), 21 n'ont pas évoqué cette question (7). On présume que toutes les patientes avaient des tumeurs porteuses de récepteurs hormonaux positifs puisque, dans les centres où elles ont été soignées, le tamoxifène en adjuvant n'était prescrit qu'en cas de tumeur RH+. Toutes les femmes interviewées ont été opérées. Après l'intervention, 10 ont subi une radiothérapie puis une hormonothérapie (tamoxifène) et 24 ont subi la séquence chimiothérapie-radiothérapie-hormonothérapie. Enfin, 4 femmes ont refusé le tamoxifène prescrit : 1 avait une tumeur de stade 1, 1 une tumeur de stade 2, 2 n'ont pas donné l'information. Deux femmes ont interrompu prématurément et définitivement la prise du tamoxifène : 1 avait une tumeur de stade 1, 1 n'a pas donné l'information.

Les femmes ont été rencontrées par l'intermédiaire de deux oncologues et d'un radiothérapeute exerçant au sein des deux centres de lutte contre le cancer investigués. Les entretiens ont été réalisés selon le souhait des femmes, soit à leur domicile, soit à l'hôpital.

Les entretiens ont repris les différents temps du parcours de soin au sein de l'institution (annonce du cancer, étape chirurgicale, chimiothérapie, radiothérapie, prescription du tamoxifène et consultations de suivi), le vécu de chacune des étapes, les autres recours thérapeutiques relatifs au cancer (conventionnels et non conventionnels), ainsi que l'impact de la maladie et des traitements sur les relations sociales et affectives. Ils ont

(7) Qui n'a pas été posée systématiquement. 
conservé la dynamique d'une conversation afin de créer une situation d'écoute telle que l'informateur et le chercheur puissent disposer d'une liberté de propos et que la personne interviewée ne se sente pas en situation d'interrogatoire (Olivier de Sardan, 1995). Pour cette étude, qui visait à comprendre les pratiques médicamenteuses des malades (Conrad, 1985) et leur suivi du traitement, nous avons plus particulièrement recherché quels savoirs populaires (représentations du corps, de la maladie, de la féminité, du médicament) sont mobilisés au quotidien par les patientes pour penser le médicament, son mode d'action, ses indications, les perceptions de son efficacité et de ses effets indésirables. Nous avons questionné la gestion quotidienne du médicament, l'expérience sociale du traitement (statut de malade, continuité du traitement, contraintes sociales et matérielles inhérentes aux traitements) et les éléments organisationnels du système de soins (fonctionnement du système de soins, modalités de suivi des patients).

Des entretiens semi-structurés ont été réalisés avec 8 médecins prescripteurs, dans les deux centres de lutte contre le cancer : 6 oncologues ( 1 femme et 5 hommes) et 2 radiothérapeutes ( 1 femme et 1 homme). Ils ont exploré leur perception de l'adhésion des femmes traitées et de l'observance, les difficultés du traitement, leur propre adhésion aux traitements prescrits. La durée des entretiens a été courte (en moyenne 30 minutes), les praticiens ayant peu de temps à accorder à la recherche en raison de leurs charges de travail. Des entretiens informels ont permis de recueillir des données complémentaires.

L'analyse présentée dans cet article s'est principalement centrée sur les entretiens, mais elle a été enrichie par les matériaux recueillis lors de l'enquête de terrain. Une analyse verticale des entretiens (par enquêtée) a permis d'esquisser les catégories thématiques prévisibles, ainsi que des dimensions émergentes (approche inductive). L'analyse des données a commencé dès les premières observations et les premiers entretiens, selon les stratégies d'analyse proposées par Glaser et Strauss (1967) reposant sur l'idée que l'analyse du matériel qualitatif est un processus continu de comparaisons qui débute à la première entrevue, se poursuit jusqu'à la fin du recueil des données et fait émerger de nouvelles catégories thématiques. Celles-ci organisent le corpus en le structurant et permettent de rassembler les éléments de l'enquête et de les analyser ensuite dans une perspective à nouveau verticale et transversale. Ainsi, dans les entretiens avec les femmes, les thèmes prévisibles sont apparus tels que ceux relatifs au vécu des effets indésirables ou à la perception de l'efficacité du traitement. D'autres thèmes comme la notion de choix, la participation à la décision médicale, la perception des risques de récidives et des risques liés au traitement, l'ambivalence du traitement ainsi que les représentations de 
la ménopause ont émergé au cours de l'analyse. Le thème de la «balance risque-bénéfice », prévisible dans les discours des praticiens, a émergé de manière récurrente dès les premiers entretiens formels et informels conduits avec ces derniers. Il nous a paru particulièrement fécond pour explorer le processus décisionnel des patientes.

Dans un premier temps, nous analyserons les perceptions des bénéfices du traitement par les femmes et leur appréhension des risques liés au traitement ou à son absence. L'objectif est de mettre en lumière les perceptions et les représentations sociales de la maladie et du médicament qui sous-tendent leur calcul des risques et des bénéfices. Dans un second temps, nous analyserons la rhétorique médicale autour de la balance risque-bénéfice et les décalages entre le discours médical et l'expérience des traitements vécue par les femmes.

\section{La balance bénéfice-risque profane}

\section{Penser l'efficacité d'un traitement}

La plupart des femmes interviewées considèrent le tamoxifène comme un traitement préventif de la récidive. Si cette perception s'accorde avec la conception biomédicale du traitement adjuvant, elle n'est cependant pas exempte d'une dimension conjuratoire de l'acte - la prise d'un médicament. Celle-ci est présente dans les entretiens, de même que dans leur dialogue avec le médecin : " c'est rassurant d'avoir un traitement », «on se raccroche à quelque chose », "ça calme l'angoisse », «je me sens protégée », "c'est une chance en plus de pouvoir prendre quelque chose », "ça te sauve la vie ». Cette dimension symbolique de la prise médicamenteuse est d'autant plus forte que les représentations sociales qui associent le cancer à un fléau et à une maladie incurable (Pinell, 1992) sont particulièrement prégnantes dans les discours des femmes interviewées.

Le bénéfice thérapeutique du tamoxifène est également pensé dans une réflexion globale sur la prise en charge de leur cancer du sein. En effet, pour celles qui adhèrent à l'ensemble du protocole de soin, le tamoxifène est appréhendé comme la partie d'un tout («le plan de traitement », «le programme thérapeutique », «le parcours personnalisé de soins ») et, lorsque leur adhésion est acquise pour ce «scénario », celle au tamoxifène l'est de fait, aucun traitement n'apparaissant plus important qu'un autre. À l'inverse, d'autres femmes opèrent une certaine hiérarchisation, résultant d'élaborations d'ordre symbolique, dans l'importance 
accordée aux différents traitements. La chirurgie apparaît ainsi comme « incontournable » (« retirer le sein c'est comme retirer la maladie »), et la chimiothérapie adjuvante primordiale, cette dernière étant, dans les représentations sociales, un traitement qui permet de "guérir » le cancer (Cannone et al., 2004 ; Rosman, 2004).

"Je ne suis pas sûre que ce traitement soit utile. Voilà. Quand j'en ai discuté avec le docteur, en fait c'est tout des statistiques : sur un certain nombre de femmes ça va marcher, sur d'autres non. Comme elle m'a dit "peut-être que vous faites partie de celles sur qui ça marche", j'ai dit : "oui, mais peut-être je fais aussi partie de celles pour qui ça ne marche pas". Elle m'a dit : "ça, je ne peux pas vous le dire”, voilà, c'est comme les chimios ça marche ou ça ne marche pas, voilà. Mais autant je ne me vois pas ne pas faire de chimio, parce que là j'aurais vraiment l'impression de ne rien faire, je ne me vois pas ne pas faire les rayons, ou la curithérapie, mais le tamoxifène... pour moi c'est pas... j'ai l'impression que ce n'est pas essentiel, je ne sais pas comment vous dire... (...) Bon alors après... c'est ce que je disais au médecin : "est-ce qu'il y a des rechutes avec tamoxifène ?", elle me disait "oui, quand même”. Bon voilà, moi c'est un truc : ou c'est sûr à $100 \%$ ou pas, mais je n'ai pas envie de passer 5 ans avec, et de toute façon après 5 ans je ne l'ai plus. Pourquoi on l'arrête dans 5 ans, pourquoi on ne continuerait pas? Si c'est vraiment efficace, après je ne l'ai plus compris ce traitement » (Julie, 39 ans, a interrompu le tamoxifène au bout d'un an).

Les doutes sur les effets bénéfiques du tamoxifène sur la prévention des récidives, et par conséquent sur son utilité, renvoient à une suspicion de «surmédicalisation» (8): "Je me suis posée la question : est-ce que c'est bien utile de prendre ça cinq ans encore ? (...) Maintenant que je vais bien, c'est plus facile de se la poser que de se dire "un traitement hormonal, c'est pas très..." Moi ça me fait un peu peur les traitements hormonaux, je me demande si on n'est pas surmédicalisé, si ils ne font pas des choses en se disant "au cas où", enfin... je ne suis pas sûre que ce soit une étape obligée... et, c'est comme je me suis posée la question pour la chimio, mais enfin c'est facile de se la poser une fois que, mais sur le moment, on se dit: "Je prends le risque que je fais pas tel ou tel traitement. Quels sont les impacts quoi ?". C'est un peu dur de jouer à la loterie, mais j'ai l'impression qu'on a tendance à trop faire des traitements, je ne suis pas sûre que ce soit très utile »(Pauline, 37 ans).

(8) L'argument de «sur-traitement » a été au cœur de la controverse sur la prévention primaire des cancers du sein par tamoxifène pour les femmes considérées à haut risque (voir Bourret et al., 1995 ; Fosket, 2004). 
Cet extrait d'entretien souligne aussi la connotation négative du tamoxifène pour certaines femmes en raison de l'analogie qu'elles font avec une « hormone» (9). En effet, les controverses autour des traitements hormonaux substitutifs de la ménopause (10), ainsi que la médiatisation des mésusages des hormones (dopage, par exemple) participent à une suspicion des usagers envers les traitements hormonaux. Dès lors, on comprend que ceux-ci soient plus difficilement pensés comme des traitements du cancer à part entière et que la perception des effets bénéfiques du tamoxifène sur la prévention des récidives puisse en être affectée.

Cependant, les extraits cités suggèrent également une appréhension du risque de récidive qui ne relève pas d'un calcul probabiliste tel que le conçoivent les professionnels. Ainsi que le souligne R. Massé (2003), le sens commun raisonne en termes binaires (absence/présence du risque). Pour certaines, derrière l'opacité des statistiques, il y a aussi la certitude de pouvoir y échapper. Le discours de Yukiko (11) est à ce titre illustratif. Cette femme de 57 ans a refusé de prendre le tamoxifène. Ce choix qu'elle qualifie de "risque calculé » s'appuie sur plusieurs facteurs, dont le recours à des médecines non conventionnelles visant à prévenir la récidive, et l'expérience d'une cousine ayant eu des effets indésirables importants. S'ajoutent ses doutes sur l'efficacité du traitement : "J'ai bien pesé le pour et le contre, mais moi, dans la balance de ne pas le prendre, c'était beaucoup plus lourd de... Le seul positif, c'est que, il paraît que, il y a moins de récidives. Mais on peut en faire une quand même, tout en souffrant pendant 5 ans (...) Donc, je me suis dit, j'aurai peut-être une récidive, mais quand même, je n'ai pas trop de risque d'en avoir une. Mais par contre, la seule chose dont je suis certaine, c'est que pendant 5 ans je vais souffrir parce que, avec mes allergies et mes intolérances aux médicaments, je vais avoir la totale. Quand je vois ce que j'ai eu avec la chimio, alors je me dis non. Je préfère avoir une récidive et ne pas avoir souffert pendant 5 ans (...) Donc, moi je pense que c'est quand même un risque calculé (...)»

C'est probablement parce que Yukiko possède un capital socioculturel élevé malgré ses faibles ressources économiques actuelles qu'elle a pu s'affirmer comme usager dans la relation médecin-malade et imposer

(9) Pour un développement du sens du tamoxifène pour les femmes et de l'analogie faite par elles avec une « hormone», voir Pellegrini et al. (2010).

(10) Pour une analyse de ces controverses, voir Löwy et Gaudillière, (2006).

(11) C'est le pseudonyme qu'elle s'est choisi. Yukiko n'est pas d'origine japonaise mais elle a vécu plusieurs années au Japon. 
sa décision à l'égard de la prise du traitement, malgré les arguments que lui ont opposés les oncologues.

Pour d'autres femmes, les avis médicaux divergents sur l'indication du tamoxifène, génèrent ou accentuent l'incertitude. Ainsi, Noémie (38 ans) s'est appuyée sur une absence de consensus médical sur son cas (à la marge de l'indication de tamoxifène puisque le bénéfice attendu est faible) pour refuser un traitement dont elle craint avant tout les effets indésirables : "Pourquoi je n'ai pas voulu prendre le Nolvadex ${ }^{\circledR}$ (12)? Alors déjà, en fin de compte, il y a deux raisons. La première, qui est quand même la plus importante on va dire, c'est... le taux, en fin de compte... alors je ne sais pas comment ils appelaient ça à l'époque, il fallait que j'aie plus de 0,5\%... je ne me rappelle plus le terme exact, mais, justement, j'étais en dessous. Donc il y avait trois docteurs. Deux étaient, on va dire mitigés pour que je le prenne, en disant "bon, je pense que c'est peut-être pas la peine", et le Pr X, lui par contre voulait à tout prix que je le prenne (...) Il ne voulait pas même pas chercher à comprendre. Il fallait le prendre! Et je suis allée contre son désir, et je me suis dit : après tout, sur trois, la majorité l'emporte ».

Les discours contradictoires des médecins amènent les femmes à réévaluer leur engagement dans une pratique médicamenteuse. De plus, ils leur donnent l'opportunité de participer de manière plus active à leur prise en charge et à la gestion de leur traitement. En effet, devant un savoir médical mal stabilisé (absence de consensus), les femmes vont se réapproprier l'expertise au travers du partage des expériences lors de discussions informelles dans les salles d'attente, mais aussi sur les forums de discussion.

\section{Se représenter les risques du traitement}

Les femmes interviewées vivant depuis plusieurs mois l'expérience du cancer et de son traitement mutilant et invalidant, le risque de cancer iatrogène ne leur paraît pas comme " une faible probabilité » (13), mais comme un danger réel auquel elles se sentent directement exposées. " $S i$ vous regardez (la notice), vous avez l'impression que vous allez vous choper un cancer de l'utérus de suite! (...) Je venais de sortir...enfin de sortir entre guillemets, parce que c'est pas sûr que je m'en sois sortie pour l'instant, mais, je venais de me taper un cancer du sein et je vois sur le

(12) Nolvadex ${ }^{\circledR}$ est un nom commercial de marque du tamoxifène.

(13) Moins de $1 \%$ selon les essais cliniques. 
tamoxifène... Ah! je n'ai retenu que ça. Ah! je n'ai retenu que ça!!!» (Michèle, 49 ans).

De plus, leur sentiment d'incertitude sur une rechute de leur maladie («l'épée de Damoclès ») transforme leur rapport au risque qui, d'un risque «faiblement probable », devient un risque «possible ». L'expérience préalable de la maladie cancéreuse et leur sentiment d'incertitude sur une rechute possible influencent l'interprétation des probabilités dans le sens d'une surestimation du risque iatrogène, constituant un biais de perception, déjà décrit dans la littérature (Rees et al., 2001), lié à un biais de disponibilité (14). Dès lors, les femmes peuvent avoir le sentiment d'avoir à « choisir entre deux cancers » et vivent l'hormonothérapie comme un «dilemme».

Devant la crainte d'être exposées au risque de développer un nouveau cancer, elles multiplient les sources d'informations, outre la « notice » jointe au médicament et lue quasi-systématiquement par les femmes : prescripteur, Internet, ouvrages de vulgarisation, réseau familial et médical, partage d'expériences avec d'autres malades. Cette recherche d'informations complémentaires les expose à des avis discordants qui peuvent augmenter le niveau d'anxiété et le coût psychologique de la prise de décision (Protière et al., 2000). Si des études montrent que, du point de vue des patients, les médecins sont la source d'information sur les traitements du cancer la plus précise et la plus sûre (Bakker et al., 2001), nos données empiriques indiquent que ce n'est pas seulement l'expertise médicale (i.e. le savoir et son application) qui est recherchée. En effet, pour plusieurs femmes, la prise en compte de la singularité de la personne et de ses affects, ainsi que la reformulation du discours médical par le prescripteur, sont nécessaires pour requalifier le risque dans des termes signifiants pour elles et l'appréhender comme étant faible : «Et puis quand en parallèle on sait que ce traitement a un risque de provoquer un cancer de l'utérus, on se dit "oui, on traite d'un coté mais c'est peut-être pour...” (...) J'ai posé la question à la personne qui me suivait (...) Elle m'a dit qu'ils estimaient qu'il y a moins de risque d'avoir un cancer de l'utérus qu'un cancer du sein, et que le cancer de l'utérus se soigne mieux que le cancer du sein. Donc (ton appuyé) ils préféraient prendre ce risque...» (Pauline, 37 ans).

(14) Un biais de disponibilité est une « erreur cognitive » qui consiste à tirer une conclusion en fonction des exemples équivalents que l'on a à l'esprit et qui sont aisément disponibles (Bronner, 2007), ici l'expérience préalable du cancer du sein des femmes interviewées. 
Dans ces cas, la parole médicale a procédé à une traduction du risque. Cela souligne l'incommensurabilité entre, d'une part, le risque perçu à travers leur expérience par les individus qui y sont exposés et, d'autre part, celui estimé par une probabilité des professionnels de santé (Lock, 1998).

\section{Perception des désavantages du traitement}

Les effets corporels de la privation œstrogénique induite par le tamoxifène sont mal vécus. Les femmes que nous avons rencontrées parlent de leur expérience du traitement au travers des nombreux effets indésirables qu'elles ressentent et qu'elles vivent péniblement parce qu'ils altèrent la qualité de vie et ont des conséquences gênantes sur la vie sociale par leurs impacts négatifs sur la sexualité, sur l'activité professionnelle (caractère gênant des bouffées de chaleur dans les rapports sociaux, fatigue, diminution de la concentration et de la performance) et sur les relations familiales (irritabilité, fatigue, baisse de la libido). Cependant, le vécu négatif des effets indésirables n'est pas forcément un facteur de déstabilisation de l'adhésion au tamoxifène. En effet, une adhésion forte au traitement peut induire en retour une minimisation ou une banalisation des effets indésirables, comme l'indique l'extrait d'entretien de Rachèle dont l'adhésion au tamoxifène, inexistante au début du traitement, s'est construite progressivement, ce qui la conduit à développer des tactiques de disculpation du médicament par la mise en cause d'autres facteurs : " Au niveau des effets secondaires...j'étais migraineuse, j'ai eu de nouveau des migraines. Est-ce que c'est le tamoxifène? Je n'en sais rien parce que pendant un an, je n'ai rien eu. Il faut dire aussi que j'ai pris le tamoxifène et j'ai repris à travailler (en même temps). Donc est-ce que les migraines sont liées au tamoxifène? À la reprise du travail où l'on me demande d'être un peu plus speed... Après, j'ai grossi, mais bon peut-être aussi parce que j'ai arrêté de fumer, depuis un an. J'ai commencé à grossir pendant la chimio aussi (...) Des bouffées de chaleur. Alors, est-ce que ça les accentue? J'en avais déjà, mais alors maintenant je suis branchée sur un radiateur en permanence (rire) Toutes les heures, d'un coup je suis en sueur, sueur, sueur! » (Rachèle, 46 ans).

Inscrits dans la vie quotidienne et les circonstances qui la caractérisent, les effets indésirables apparaissent pour les personnes comme des 
aléas de la vie de tous les jours. Ce processus de déplacement de la cause des symptômes permet de minorer la responsabilité du médicament (15).

À la réalité biologique (la gêne occasionnée par la privation œstrogénique) s'ajoutent une perception et une reformulation du tamoxifène comme un médicament «ménopausant » et «vieillissant 》. "Moi je souhaitais absolument, dès que je serais ménopausée, faire le traitement substitutif, parce que je me suis dit: "Je vais avoir plein de rides ; je vais avoir une peau : ça va être affreux, je vais vieillir ; je vais grossir”. Et puis le traitement substitutif, il n'en a pas été question parce qu'il y a eu le cancer du sein, et donc, je n'ai pas eu tellement de temps... J'ai été ménopausée, j'ai commencé le tamoxifène... je me suis dit : ça y est là, j'ai le pied dans, quelque part dans la vieillesse » (Christina, 56 ans ; 52 ans lors de la découverte du cancer).

Le tamoxifène est réinterprété au travers des représentations de la ménopause. Or, dans les sociétés occidentales, l'image de la femme ménopausée est associée au déclin biologique, symbolique et social (perte de la féminité et du pouvoir de séduction, perte du statut lié à l'arrêt de la fonction de reproduction, perte du capital santé) (Delanoë, 2006). Du fait de cette représentation sociale déficitaire de la femme ménopausée et des enjeux psychiques relatifs à la féminité, les effets corporels de la privation œstrogénique induite par le tamoxifène seront d'autant plus mal vécus que les femmes sont jeunes et/ou non ménopausées au début du traitement (16). De plus, toutes les patientes ont traversé une phase au cours de laquelle leur image corporelle a été malmenée principalement par la perte des cheveux et des poils, et par l'amputation pour celles qui ont subi une mastectomie (17). L'image - féminine et esthétique - de soi est en cours de reconstruction lorsque le traitement par tamoxifène est instauré, d'où la difficulté à accepter qu'elle soit à nouveau altérée.

(15) On observe la même tendance à minimiser et à banaliser les effets indésirables dans les forums sur Internet, où les réponses aux questions parfois angoissées des femmes trouvent des réponses rassurantes auprès des internautes connaissant le même parcours de soin. Leurs messages associent deux registres : un registre pratique (description des effets avec banalisation, échanges de «trucs » pour les atténuer), un registre de soutien avec des encouragements à poursuivre le tamoxifène et des messages d'espoir à destination de l'internaute qui a posé la question et de toutes celles qui liront le message.

(16) Dix femmes étaient âgées de moins de 45 ans et non ménopausées au moment du diagnostic.

(17) Mais elles subissent aussi d'autres modifications de l'aspect corporel qui sont plus ou moins bien tolérées telles que la prise de poids, la modification de l'aspect de la peau ou des cheveux, etc. 


\section{«On n'a pas le choix»}

Le postulat de la rationalité utilitariste implique que, à l'issue du processus cognitif évaluant les risques et les bénéfices, les individus expriment un choix et prennent une décision. Or, un grand nombre de femmes interrogées ont utilisé une même formulation - «on n'a pas le choix » - pour parler de leur rapport aux prescriptions médicales. Bien souvent, cette déclaration traduit le fait que, face à une maladie qui met en jeu le pronostic vital, la question d'un traitement alternatif à celui qui est proposé ne se pose même pas.

Dans d'autres cas, l'expression «on n'a pas le choix » soulève la question de la participation des patientes aux décisions médicales. Elle signifie alors « on ne nous laisse pas le choix » et renvoie à des positions différenciées selon l'histoire et la structuration psychique de chaque femme :

- la résignation d'emblée à prendre le traitement : le choix entre un type de traitement et «ne rien faire » n'est pas perçu comme un choix par les femmes qui considèrent que la seule possibilité offerte est de prendre le traitement proposé (Charles et al., 1998 ; Elit et al., 2003). L'absence de traitement n'est pas envisagée comme une option et les femmes ne pensent pas avoir à prendre part à la décision thérapeutique les concernant. Les propos de ces patientes illustrent cette position : «Donc j'ai pris le tamoxifène religieusement pendant 5 ans (...) J'ai pensé que je n'avais pas le choix » (Simone, 56 ans) ; «Ah non, là, il n'y avait pas le choix (Ils vous ont dit qu'il n'y avait pas le choix ?) Ah! Il fallait le prendre. Ah oui, ça, il fallait le prendre » (Christina, 56 ans) ;

- l'adhésion au projet thérapeutique dans une relation asymétrique d'obéissance à l'égard du médecin fondée sur la confiance dans le praticien ou dans l'institution : la malade n'envisage pas, voire refuse, la possibilité de prendre part à la décision thérapeutique la concernant, ne s'accordant ainsi aucune marge d'autonomie. L'extrait d'entretien suivant en est exemplaire : "Oui, j'avais le choix : ou je le prenais, ou je ne le prenais pas. Alors... bon, j'ai fait un choix, je dirais un peu au hasard: "Est-ce que je le prends? Est-ce que je ne le prends pas?" Je dirais que j'ai entièrement confiance en monsieur M. (oncologue) et je me suis dit que ça avait l'air de lui tenir tellement à coeur que je le prenne, je me suis dit qu'il y a certainement un bien fondé quelque part. Je me suis dit : "Il a fait des études"... S'il a décrété que c'était bon pour moi, je vais le prendre » (Sarah, 46 ans);

- l'acceptation du traitement mais avec le regret d'une absence de négociation dans une relation médecin-malade perçue comme asymétrique : la patiente souhaiterait une plus grande participation à la décision théra- 
peutique mais sans contester l'expertise et l'autorité médicale : "Il est vrai que vous entrez dans un système, vous êtes prise en charge totalement, on ne vous laisse pas le temps de la réaction et, peut-être que ça dépend des individus, moi je sais que j'ai toujours été assez indépendante, assez autonome, et le fait qu'on ne me laisse pas de choix, si vous voulez, m'a énormément perturbée. Parce que quand vous avez quelqu'un qui vous dit "Mais, c'est obligé, tout ça, qui vous dit oui, oui, oui, vous faites ça, ça, ça”, on ne vous laisse pas le temps de la réflexion, on ne vous laisse pas souffler! » (Maryse, 49 ans) ;

- le refus d'une position passive perçue comme assignée à la patiente par le corps médical : ces femmes veulent se sentir libres d'accepter ou non le traitement proposé et donc prendre la responsabilité de leur propre vie. Elles critiquent la dépendance au monde médical imposée par la prise en charge actuelle du cancer. Elles manifestent leur opposition à ce qu'elles perçoivent comme une perte de liberté et une déresponsabilisation par de multiples reproches, doléances ou contestations des traitements, qu'elles adressent aux soignants. Elles expriment leur volonté d'être actrices à part entière de leur traitement en le refusant ou en l'interrompant, d'une part, et par le recours aux médecines non conventionnelles, d'autre part : "C'est vrai que la chirurgie, je n'ai pas eu le choix, la chimio je n'ai pas eu le choix, les rayons je n'ai pas eu le choix, alors c'était un peu... l'hormonothérapie... enfin... la chimio je savais que c'était imposé, je ne pouvais pas... enfin il ne m'est pas venu à l'esprit de dire "Non, je ne veux pas de chimio". Mais l'hormonothérapie, enfin je pouvais dire "Je refuse”...» (Coralie, 35 ans).

Le refus du tamoxifène est une issue pour résoudre le dilemme entre l'obligation morale de prendre un traitement contre le cancer et le besoin d'autonomie socialement valorisé dans le champ de la santé (Moulin, 2004). Cependant, le refus du traitement n'est pas la seule voie d'autonomisation. D'autres femmes qui le poursuivent ont pris une part active à leur prise en charge en choisissant leur parcours de soin, en multipliant les avis diagnostiques et en refusant d'être suivies par certains médecins. Elles revendiquent aussi le principe du consentement éclairé aux soins et la possibilité de choisir de poursuivre ou non le traitement par tamoxifène après avoir été informées des risques et des bénéfices.

\section{Rhétorique médicale sur la balance risque-bénéfice}

Aux paramètres ayant trait à l'expérience individuelle et sociale du traitement, ou ayant une dimension d'ordre symbolique, et qui participent 
à la décision de débuter un traitement puis de le poursuivre, s'ajoute l'information transmise par l'oncologue. Celle-ci influencerait significativement la décision du patient (Chao et al., 2003), mais sous quelle forme est-elle transmise?

La proposition de traitement du tamoxifène en adjuvant n'émane pas d'un seul médecin. Elle est émise lors d'une réunion de concertation pluridisciplinaire (RCP) (18). La recommandation prend en compte, dans la balance décisionnelle, uniquement les standards fondés sur la diminution du taux de récidive de cancer du sein, et non la qualité de vie des malades. Dans l'un des centres où nous avons conduit nos observations, le sujet et son corps sont réduits aux caractéristiques histologiques de la tumeur lors des discussions sur le type de chirurgie, sur la séquence des traitements et le type de traitement adjuvant. Les conditions de réalisation de ces RCP (très grande quantité de dossiers à traiter en un temps réduit, à la fin d'une longue journée de travail pour tous les médecins présents) expliquent en partie - que les professionnels resserrent leurs réflexions autour de points techniques, écartant ainsi tout risque de voir leur expertise brouillée par l'irruption du sujet dans la décision médicale. Cette distanciation contraste avec l'observation de RCP dans un autre centre où des caractéristiques comme l'âge physiologique de la personne, le volume et la forme de sa poitrine, voire parfois (mais rarement) ses caractéristiques sociales ou psychologiques, sont convoquées dans la décision thérapeutique.

L'information sur les avantages et les désavantages du tamoxifène est donnée lors de la consultation qui marque la fin des traitements hospitaliers (chimiothérapie et/ou radiothérapie). Les médecins interviewés déclarent s'appuyer sur leur expérience et leur savoir-faire pour appréhender au mieux la demande d'informations des femmes sur les bénéfices thérapeutiques. Ils disent adapter leur discours, d'une part, au niveau d'anxiété et aux attentes qu'ils perçoivent chez chaque femme et, d'autre part, aux capacités de compréhension de la malade qu'ils supposent. Cette manière de procéder s'appuie sur des critères sociocognitifs subjectifs en l'absence de connaissance réelle de la biographie du patient, ainsi que l'a déjà noté S. Fainzang (2006). Elle se retrouve dans plusieurs études qui ont montré que les caractéristiques personnelles des malades, comme le niveau d'éducation, influencent la communication du médecin (Street, 1991), l'information donnée étant quantitativement proportionnelle au niveau socioculturel du patient (Martinez, 2005 ; Waitzkin, 1991).

(18) Sur le fonctionnement des RCP et la diffusion de l'approche collective (entre pairs) de la décision médicale, voir Castel (2008). 


\section{Des récits prédictifs}

Les bénéfices thérapeutiques sont exprimés en termes de probabilités avec une grande hétérogénéité selon les médecins, comme le montrent ces extraits d'interviews d'oncologues : "Je donne une fourchette de risque de rechute locale ou controlatérale »; "Je dis que ça divise le risque de récidive par deux ou par trois »; "Pour le sein controlatéral, ça élimine quand même environ $60 \%$ »; «10\% de bénéfice absolu (...) Je leur dis : il suffit que ça tombe sur vous, et comme je ne peux pas savoir, on l'a généralisé »; "Grosso modo, pour les femmes qui ont une hormonosensibilité, je leur dis que les chiffres actuels, c'est environ $30 \%$ de risque de récidive »; "On leur explique que ça diminue grandement (ton appuyé) le risque de rechute. Souvent, on leur explique que ça diminue le risque de rechute de 30 à $50 \%$ ». Cette disparité des réponses traduit la complexité de l'information médicale à transmettre concernant la réduction attendue du risque de récidive, selon que l'on considère le risque absolu (probabilité qu'une récidive survienne sans traitement adjuvant) ou le risque relatif (comparaison du risque de survenue d'une récidive avec ou sans traitement adjuvant), la différenciation entre risque absolu et risque relatif n'étant pas toujours bien comprise par les cliniciens (Loprinzi et Thomé, 2001).

Elle montre aussi que les récits cliniques - i.e. les histoires thérapeutiques élaborées par les médecins, pour et avec leurs patients, au sujet de la poursuite du traitement et de l'évolution de la maladie (DelVecchio Good et al., 1994) - construits autour des statistiques visent à transmettre de la certitude par l'anticipation de bénéfices thérapeutiques, là où règne l'incertitude. Ces stratégies narratives sont similaires à celles de leurs collègues américains (DelVecchio Good, 1995).

De plus, l'observation des consultations montre que les médecins présentent le tamoxifène comme un élément favorable, un «plus » pour la patiente, voire une « chance ». La rhétorique médicale lors de la consultation (" traitement de prévention », "par (ou de) sécurité », "précaution », «protection », " pour réduire le risque ») permet d'évoquer la possibilité d'une rechute tout en assurant la patiente que tout est fait pour la protéger. En effet, comme l'a montré M. Ménoret (2007), le traitement médical de l'incertitude repose sur l'engagement à mobiliser toutes les ressources disponibles.

Percevant ou anticipant les difficultés des patientes à comprendre l'information et à se l'approprier, les médecins adaptent leur discours et font usage de stratégies narratives visant à transformer une probabilité en une catégorie ayant, de leur point de vue, plus de sens pour les femmes : 
- la transformation du pourcentage en nombre : «Je leur dis : finalement le tamoxifène, à qui ça sert? Si on prend maintenant tous les cancers dans le monde, ça sauverait combien de femmes? Et quand je leur dis le chiffre... environ 2000 , là elles disent "Ah oui, c'est important" (...) ça les rassure d'avoir le poids de l'effet du médicament » (oncologue, 51 ans);

- le renforcement des chiffres afin d'accentuer l'impression de bénéfices thérapeutiques attendus : «Les $50 \%$ de rechutes évitées par rapport à un chiffre qui est beaucoup plus petit, mais enfin, grosso modo, on est, je pense, assez malhonnête pour leur dire 30 à $50 \%$ de récidives en moins par rapport à ce qui doit arriver mais pas par rapport à un nombre total que, elles, elles imaginent » (oncologue, 36 ans) ;

- la singularisation : "J'axe le problème sur le risque attaché à leur (ton appuyé) maladie. Donc en général, j’axe le discours sur les facteurs pronostiques de chaque personne et non pas le cancer du sein de tout le monde » (oncologue, 40 ans) ;

- l'élimination de la question des chiffres : «Ce n'est pas tellement les chiffres qu'elles veulent. Ce n'est pas ça qui les intéresse. Ce qu'elles veulent savoir, c'est les effets secondaires » (oncologue, 44 ans).

L'effort pédagogique des médecins est déjà un mode d'interprétation des données scientifiques qui engage nécessairement et logiquement la subjectivité de chacun. Cependant, à l'instar de l'épidémiologie qui construit des « récits prédictifs » traduisant des estimations en termes de menaces ou de dangers, et qui sont façonnés par des a priori culturels (Massé, 2007), les médecins interviewés construisent leur récit prédictif du risque de rechute en conjuguant l'incertitude inhérente à la cancérologie avec leurs normes et valeurs professionnelles. Parmi celles-ci, la «prédilection pour l'action 》 (i.e. «l'action comme une fin en soi, en partant de l'hypothèse bâtarde qu'il vaut mieux faire quelque chose que de rien faire du tout »), à l'origine de la propension à la prescription médicamenteuse (Friedson, 1984 : 177), est une norme professionnelle qui légitime l'intervention médicale.

\section{Banalisation des effets indésirables}

La plupart des médecins interviewés considèrent que la tolérance au tamoxifène est «plutôt pas mauvaise » ou «plutôt bonne » parce qu'ils la comparent aux effets secondaires de la chimiothérapie. Ils minimisent ainsi les bouffées de chaleur ou la prise de poids - «gène modérée », "ce n'est pas un problème de prendre du poids parce que ce qui les (femmes) intéresse avant tout, c'est de ne pas rechuter » (oncologue, 44 ans en 
entretien). En l'absence de réponse médicamenteuse efficace, ils ont tendance à écouter les plaintes des femmes sans y répondre - " $c$ 'est normal » (oncologue, 40 ans, en consultation) - , ou à répondre en termes d'hygiène de vie. Ils peuvent alors donner l'impression aux femmes de sous-estimer les symptômes avec des discours fatalistes, les invitant à la résignation - «il faut accepter ces petits inconvénients » (radiothérapeute, 58 ans, en consultation). D'ailleurs, certaines femmes nous ont confié ne plus aborder le thème des effets indésirables lors des consultations car elles estiment que les médecins hiérarchisent la gravité des effets secondaires et banalisent les effets indésirables de la privation oœstrogénique. De plus, la mauvaise tolérance est parfois rapportée par les médecins à une cause " psychologique » : autosuggestion (par la lecture de la notice), dépression, manifestation d'une agressivité. Cette tendance à la psychologisation, voire à la psychopathologisation observée dans d'autres travaux (Fainzang, 2006), les dispense d'une prise en compte de la qualité de vie des patientes.

Nous observons donc un décalage entre la perception des effets secondaires et leur pénibilité par les médecins et par les femmes (19). Nous l'expliquons, à l'instar d'autres auteurs (Ford et al., 1996), par le peu d'espace de discussion accordé au vécu psychosocial d'une situation clinique, que nous avons constaté au cours de l'observation des consultations. Les charges de travail des médecins dans les centres où a été conduite l'étude (consultations surchargées, activité dans les services, activité de recherche clinique, RCP, réunions administratives) leur laissent peu de temps pour un suivi des patientes qui accorderait une large part à l'écoute psychosociale et qui prendrait en compte la qualité de vie et les préférences des femmes dans la décision thérapeutique. S'ils don-

(19) Décalage que l'on retrouve également lorsque l'on demande aux médecins quels sont les effets secondaires les plus fréquents rapportés par leurs patientes. S'ils citent tous les bouffées de chaleur en première position, leurs avis divergent dans l'énumération des autres effets indésirables rapportés par leurs patientes : la prise de poids (5 médecins/8), la sécheresse vaginale (5/8), les troubles digestifs $(3 / 8)$, la sécheresse cutanée et les cheveux cassants (2/8), les troubles de la libido (2/8), les douleurs pelviennes $(1 / 8)$, les douleurs articulaires $(1 / 8)$, les troubles des règles $(1 / 8)$, la pilosité $(1 / 8)$. Ces chiffres n'ont aucune valeur statistique, ils montrent seulement que, pour une même population de professionnels (spécialisés dans le traitement et la surveillance du cancer du sein, exerçant dans des centres de lutte contre le cancer) et prenant en charge une même population de patientes (même recrutement, distribution aléatoire des patientes selon les praticiens), on observe une variabilité de l'estimation de la tolérance du médicament. 
nent des informations sur les effets indésirables du traitement, les cliniciens accordent un espace restreint à l'expression du vécu des effets indésirables, et nous avons observé une tendance à déléguer cette écoute à d'autres intervenants (médecin traitant, psychologues).

Le décalage provient aussi d'une hiérarchisation différentielle des priorités : celles-ci sont d'ordre essentiellement sanitaire pour les médecins, ce qui les fait considérer prioritairement la prévention de la récidive aux dépens de la qualité de vie, alors qu'elles sont psychosociales pour les femmes (image de soi, enjeux liés à la féminité et à la maternité, vie quotidienne et professionnelle).

Cependant, et dans la limite de l'effectif des médecins interviewés ( 2 femmes, 6 hommes), l'analyse des entretiens met en évidence une différence entre hommes et femmes dans la perception de la nature des effets secondaires rapportés par les patientes, ainsi que de leur importance ou leur impact au plan psychique. Les hommes médecins n'évoquent pas les problèmes d'ordre sexuel, ou lorsqu'ils le font, c'est sans s'y attarder : " Il y a des bouffées de chaleur, les effets de prise de poids, il y a les effets sur la sexualité, aussi. Surtout la prise de poids et les effets articulaires, on n'en parle peut-être pas assez systématiquement parce qu'avant on n'en parlait jamais » (oncologue, homme, 51 ans) ; "Bouffées de chaleur, prise de poids. Parfois des sécheresses vaginales par exemple, voilà, c'est un problème de ménopause. Mais prise de poids, ça, c'est assez fréquent la prise de poids » (radiothérapeute, homme, 58 ans).

En revanche, les deux femmes médecins y accordent une plus grande attention : "Il y a celles qui sont relativement jeunes et qui ne veulent pas avoir de troubles de la libido 》 (radiothérapeute, femme, 41 ans) ; «Elles parlent de la sexualité, mais ça dépend, parce que des fois elles viennent avec leurs maris. Alors quand elles viennent seules, elles ont quand même une baisse de libido » (oncologue, femme, 33 ans). Elles semblent aussi plus sensibles aux modifications de l'image corporelle exprimées par leurs patientes, évoquant avec compassion la souffrance induite par les transformations du corps dues au tamoxifène : "Il y en a qui voient une dégradation de leur corps. Elles disent : "Je ne ressemble plus à rien", "Qu'est-ce que je suis devenue?” ( (oncologue, femme, 33 ans).

Il est possible qu'une relation particulière se noue entre femmes au sujet des questions liées à la sexualité ou à l'intimité physique, qui peut s'expliquer par une plus grande attention portée à ces questions par les femmes médecins, ou par une plus grande propension des patientes à en parler à un médecin-femme, les deux termes de l'alternative, loin d'être exclusifs l'un de l'autre, pouvant être en jeu. 


\section{Conclusion}

Notre étude visait à documenter empiriquement le processus décisionnel concernant la prescription d'un médicament dont l'efficacité - au niveau individuel - reste incertaine et à resituer le sens du risque de prendre ou de ne pas prendre le médicament dans le contexte expérientiel, social et culturel des consommatrices. Pour une personne, un risque n'est pas acceptable ou inacceptable dans l'absolu, mais doit toujours être resitué dans ce contexte. Différents éléments interviennent dans la réinterprétation des risques (de récidive et liés au traitement) et des bénéfices attendus du médicament. L'expérience préalable de la maladie cancéreuse modifie la perception du risque iatrogène. Parallèlement, l'aspect symbolique intervient à travers la dimension conjuratoire du médicament, la hiérarchisation des traitements proposés effectuée par les patientes, ou encore l'analogie faite avec d'autres traitements hormonaux chargés de sens.

Nos matériaux soulignent les décalages entre l'appréhension des risques et des bénéfices du traitement par les patientes et les médecins. Ces derniers obéissent à une logique professionnelle d'intervention dirigée préférentiellement vers la prévention de la récidive. Cette logique sous-tend leur anticipation des bénéfices et les stratégies narratives qu'ils déploient pour mettre en récit l'information à délivrer aux patientes et qui les amènent à nuancer leurs propos en fonction de chaque situation, ce qui modifie inévitablement l'objectivité factuelle de leur discours, alors même qu'ils devraient délivrer théoriquement les informations scientifiques les plus objectives possibles. Ce faisant, ils reproduisent l'asymétrie de savoir et de pouvoir qui structure profondément la relation médecin-malade dans la société française.

Si cette logique d'intervention sous-tend également la prise en compte des effets secondaires - banalisés dans le discours des médecins - dans la qualité de vie des patientes, le poids des facteurs institutionnels n'est pas négligeable. Ainsi, l'organisation de la cancérologie institutionnelle - avec ses usages en matière de consultations de suivi privilégiant un suivi alterné aux dépens d'une relation singulière avec un médecin référent ; les charges et cadences de travail des médecins - restreint l'espace d'écoute que devraient pouvoir trouver les usagers pour exprimer leurs incertitudes sur les bénéfices et les inconvénients des traitements, mais aussi sur ce qui fait leur vie avec la maladie.

Conflit d'intérêts : aucun. 


\section{RÉFÉRENCES BIBLIOGRAPHIQUES}

Bakker D.A., Fitch M., Gray R., Reed E., Bennett J., 2001, Patient-health care provider communication during chemotherapy treatment: the perspectives of women with breast cancer, Patient Education and Counseling, 43, 61-71.

Bourret P., Eisinger F., Moatti J.P., 1995, Chimioprévention médicalisée des cancers. Un point de vue des sciences sociales, Bulletin du Cancer, 82, Suppl. $3,224 \mathrm{~s}-229 \mathrm{~s}$.

Bronner G., 2007, L'empire de l'erreur. Éléments de sociologie cognitive, Paris, PUF, Coll. Sociologies.

Cannone P., Dany L., Dudoit E., Duffaud F., Salas S., Favre R., 2004, Étude des représentations sociales de la chimiothérapie : une voie d'analyse des relations entre patients et médecins oncologues, Bulletin du Cancer, 91, 3, 279-84.

Castel P., 2008, La gestion de l'incertitude médicale : approche collective et contrôle latéral en cancérologie, Sciences Sociales et Santé, 26, 1, 9-31.

Chao C., Studts J.L., Hadley T., Roetzer L., Dineen S., Lorentz D., Youssef Agha A., McMasters K.M., 2003, Adjuvant chemotherapy for breast cancer: how presentation of recurrence risk influences decision-making, Journal of Clinical Oncology, 21, 23, 4299-4305.

Charles C., Redko C., Whelan T., Gafni A., Reyno L., 1998, Doing nothing is not a choice: lay constructions of treatment decision-making among women with early-stage breast cancer, Sociology of Health and Illness, 20, 71-95.

Clarke R., Leonessa F., Welch J.N., Skaar T.C., 2001, Celular and molecular pharmacology of antioestrogen action and resistance, Pharmacological Reviews, 53, 1, 25-71.

Conrad P., 1985, The meaning of medications: another look at compliance, Social Science and Medicine, 20, 1, 29-37.

Delanoë D., 2006, Sexe, croyances et ménopause, Paris, Hachette Littérature. DelVecchio Good M.J., Munakata T., Kobayashi Y., Mattingly C., Good B.J., 1994, Oncology and narrative time, Social Science and Medicine, 38, 6, 855862.

DelVecchio Good M.J., 1995, Cultural studies of biomedicine: an agenda for research, Social Science and Medicine, 41, 4, 461-473.

Desclaux A., Lévy J.J., 2003, Culture et médicaments. Ancien objet ou nouveau courant en anthropologie médicale ?, Anthropologie et Sociétés, 27, 2, $5-21$. 
Early Breast Cancer Trialists' Collaborative group (EBCTCG), 2005, Effects of chemotherapy and hormonal therapy for early breast cancer on recurrence and 15-year survival: an overview of the randomised trials, The Lancet, 365, 9472, 1687-717.

Elit L., Charles C., Gold I., Gafni A., Farell S., Tedford S., Dal Bello D., Whelan T., 2003, Women's perceptions about treatment decision making for ovarian cancer, Gynecologic Oncology, 88, 89-95.

Fainzang S., 2006, La relation médecins-malades : information et mensonge, Paris, PUF, Coll. Ethnologies.

Ford S., Fallowfield L., Lewis S., 1996, Doctor-patient interactions in oncology, Social Science and Medicine, 11, 1511-1519.

Fosket J., 2004, Constructing "high-risk women": the development and standardization of a breast cancer risk assessment tool, Science, Technology, \& Human Values, 29, 3, 291-313.

Friedson E., 1984 (1970), La profession médicale, Paris, Payot.

Glaser B.G., Strauss A., 2010 (1967), La découverte de la théorie ancrée. Stratégies pour la recherche qualitative, Paris, Armand Colin, Coll. Individu et Société.

Lock M., 1998, Breast cancer: reading the omens, Anthropology Today, 14, 4, 7-16.

Loprinzi C.L, Thomé S.D, 2001, Understanding the utility of adjuvant systemic therapy for primary breast cancer, Journal of Clinical Oncology, 19, 4, 972-979.

Löwy I., Gaudillière J.P., 2006, Médicalisation de la ménopause, mouvements pour la santé des femmes et controverses sur les thérapies hormonales, Nouvelles Questions Féminines, 25, 2, 48-65.

Massé R., 1997, Les mirages de la rationalité des savoirs ethnomédicaux, Anthropologies et Sociétés, 21, 1, 53-72.

Massé R., 2003, Éthique et santé publique. Enjeux, valeurs et normativité, Québec, Presses de l'université Laval.

Massé R., 2007, Épidémiologie prédictive et divination, In : Rossi I., ed., Prévoir et prédire la maladie. Divination et pronostic, Paris, Aux Lieux d'Être : 87-119.

Martinez R., 2005, "What's wrong with me ?": cervical cancer in Venezuela - Living in the borderlands of health, disease, and illness, Social Science and Medicine, 61, 797-808.

Ménoret M., 2007, Informer mais convaincre : incertitude médicale et rhétorique statistique en cancérologie, Sciences Sociales et Santé, 25, 1, 33-54. 
Moulin A.M., 2004, Ordre et désordre dans le champ de la santé, In : Schweyer F.X., Pennec S., Cresson G., Bouchayer F., eds, ,Normes et valeurs dans le champ de la santé, Rennes, Éditions ENSP, 19-36.

Olivier de Sardan J.P., 1995, La politique du terrain. Sur la production des données en anthropologie, Enquête, 1, 71-109.

Olivier de Sardan J.P., 2008, La rigueur du qualitatif. Les contraintes empiriques de l'interprétation socio-anthropologique, Louvain-La-Neuve, Academia-Bruylant.

Pellegrini I., Sarradon-Eck A., Ben Soussan P., Lacour A.C., Largillier R., Tallet A., Tarpin C., Julian-Reynier C., 2010, Women's perceptions and experience of adjuvant tamoxifen therapy account for their adherence: breast cancer patients' point of view, Psycho-Oncology, 19, 5, 472-479.

Pinell P., 1992, Naissance d'un fléau. Histoire de la lutte contre le cancer en France (1980-1940), Paris, Métailié.

Protière C., Viens P., Genre D., Cowen D., Camerlo J., Gravis G., Alzieu C., Bertucci F., Resbeut M., Maraninchi D., Moatti J.P., 2000, Patient participation in medical decision-making: a french study in adjuvant radio-chemotherapy for early breast cancer, Annals of Oncology, 11, 39-45.

Rees G., Fry A., Cull A., 2001, A family history of breast cancer: women's experiences from a theoretical perspective, Social Science and Medicine, 52, 9, 1433-1440.

Rosman S., 2004, Cancer and stigma: experience of patients with chemotherapy-induced alopecia, Patient Education and Counseling, 52, 333-339.

Street R.L., 1991, Information-giving in medical consultations: the influence of patients' communicative styles and personal characteristics, Social Science and Medicine, 32, 541-548.

Waizkin H., 1985, Information giving in medical care, Journal of Health and Social Behavior, 26, 81-101. 


\section{ABSTRACT}

\section{Balancing risks and therapeutic benefits. About adjuvant tamoxifen treatment for breast cancer patients}

One of the main tools available for making bio-medical decisions about the best treatment to prescribe is the risk-benefit ratio. This article takes a contextual approach to the decision-making processes involved in the prescription of adjuvant tamoxifen as a mean of reducing the risk of relapse of breast cancer. Using anthropological descriptive methods, it examines the discrepancy between patients' and doctors' perceptions of the risks and benefits of the treatment and analyses their underlying logics. Representations of women are related to cancer (fear of the disease), its treatment (efficacy) and tamoxifen (fear of hormones and aging), and can lead to a symbolic hierarchy of treatments, whereas doctors' professional practices focus mainly on the prevention of the recurrence of cancers.

\section{RESUMEN}

\section{Balance de riesgos y beneficios terapéuticos : el ejemplo del tratamiento auxiliar del cáncer de seno con tamoxifeno}

Uno de los elementos fundamentales para decidir una terapia es el balance riesgo-beneficio. Este articulo aborda el proceso de toma de decisiones que afecta al tratamiento de pacientes con cáncer de seno. A partir de una perspectiva antropológica descriptiva, analizamos la prescripción de un tratamiento con tamoxifeno para reducir el riesgo de recidiva. Este trabajo explora el desajuste entre las percepciones de las pacientes y de los médicos de los riesgos y beneficios del tratamiento, y analiza sus lógicas cognitivas, simbolicas y sociales subyacentes. El articulo analiza las representaciones de las mujeres relativas al cancer (miedo a la enfermedad), a sus tratamientos (eficacia) y al tamoxifeno (miedo a las hormonas $\mathrm{y}$ al envejecimiento) que llevan a una jerarquizacion simbolica de los tratamientos. Los médicos siguen una lógica profesional de intervención que privilegia la prevención de la recidiva. 